\title{
Sobrepeso e integración económica en México
}

Overweight and Economic Integration in Mexico

\author{
Eduardo Loría y Emmanuel Salas*
}

Nada es veneno, todo es veneno: la diferencia está en la dosis

Paracelso

\section{Resumen}

La obesidad en México y el mundo ha ido creciendo sistemáticamente, al grado que se ha convertido en una pandemia con fuertes consecuencias en padecimientos muy graves de salud pública como hipertensión, enfermedades cardiovasculares, diabetes y cáncer, todo lo cual tiene fuertes impactos en las finanzas públicas y en el crecimiento económico.

En México, desde los años noventa, la obesidad y el sobrepeso han crecido de manera exponencial, mucho más que en Estados Unidos. A manera de hipótesis, sugerimos que la entrada en vigor del Tratado de Libre Comercio de América del Norte en 1994 no sólo modificó la estructura del comercio y de los flujos de capital. También gestó un cambio sustancial en los hábitos de consumo de la población, particularmente la infantil, que se manifestó en un incremento notable en el consumo de golosinas. Estos resultados emergen del análisis de la Encuesta Nacional Ingreso Gasto de los Hogares (ENIGH) para los años 1992 y 1996.

\section{Palabras clave: \\ - Integración económica \\ - México \\ - Prevalencia \\ - Sobrepeso y obesidad}

\section{Abstract}

The obesity in Mexico and the world has been growing systematicly, to the degree that has turned into a pandemic with strong consequences into very serious sufferings of public health as hypertension, cardiovascular diseases, diabetes and cancer, everything which has strong impacts in the public finance and in the economic growth. In Mexico, from the nineties, the obesity and the overweight have grown in an exponential way, much more than in The United States. Like hypothesis, we suggest that the entry into force of the North American Free Trade Agreement in 1994 not only modified the structure of the trade and of the capital flows. Also it prepared a substantial change in the habits of consumption of the population, particularly the infantile one, which demonstrated in a notable increase in the consumption of delicacies. These results emerge of the analysis of the National Survey I deposit Expense of the Homes (ENIGH) for the year 1992 and 1996.

\section{Keywords: \\ - Economic Integration \\ - Mexico \\ - Prevalence \\ - Overweight and Obesity}

* Centro de Modelística y Pronósticos Económicos (CEMPe), Facultad de Economía, unAm, Edificio B, tercer piso, cubículo 305. Circuito Interior s/n, Cu, México, Distrito Federal. C.P. 04510. Teléfono: 56222142 y 56222143. Agradecemos el financiamiento al proyecto de investigación PAPIIT IN302514, México: crecimiento, ciclos y precarización laboral, 1980-2020, DGAPA, unAM. Autor principal: eduardol@unam.mx y salas.emmanuel@gmail.com. Agradecemos la asistencia de Tania Sánchez, Karen Maldonado. Leyda Sánchez, Eduardo Becerril y Malinalli Aupart. Los autores son los únicos responsables de lo que aquí se dice o se omite. 


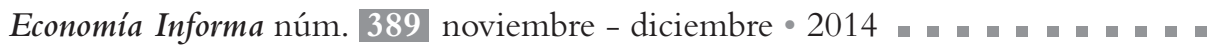

que lo ha llevado a ser el número uno en este problema a nivel mundial (ops, 2014), ${ }^{1}$ tanto entre niños como en adultos.

Tal y como lo presenta la oms (2014), la obesidad es un problema multifactorial que entre otras muchas causas tiene que ver con el sedentarismo (Hernández et al, 1999) y el estilo de vida. Sin embargo, aquí sólo lo asociaremos al desequilibrio entre las calorías ingeridas y consumidas (Rivera et al, 2012) provocado por un cambio en los factores ambientales (Booth et al, 2005) principalmente asociados a la liberalización comercial, sólo tomaremos el cambio estructural en el gasto de consumo de las familias que permite detectar la ENIGH en dos años de contraste (1992 y 1996). Consideramos que la entrada en vigor del Tratado de Libre Comercio de América de Norte en 1994 incrementó el desequilibrio calórico que ayudaría a explicar el inusitado aumento del problema de interés en México.

Castañeda (2014) ha propuesto que los cambios que México experimentó con la firma del tratado no se restringieron al ámbito financiero y comercial, sino que también hacia la esfera social, como en el caso de la conciencia democrática y los hábitos alimenticios.

La instalación de franquicias de comida rápida con altos contenidos de grasas y calorías es un factor importante a recalcar. Jeffery (2006) destaca el efecto de la introducción de franquicias de comida rápida. Young y Nestle (2002 y 2006) proponen que el incremento de las porciones ha sido un condicionante del aumento de la obesidad y del sobrepeso. Adicionalmente al tamaño de las porciones ${ }^{2}$ la composición de la nueva dieta predispone al sobrepeso al contener grasas saturadas y carbohidratos refinados (Gazzaniga y Burns, 1993).

Los cambios de estilos de vida en Estados Unidos, derivados del efecto demográfico de los baby boomers han sido destacados por varios autores. Por ejemplo, Cutler et al (2003) y Rashad et al (2005) mencionan que desde la década de 1960 ha incrementado la talla de los estadounidenses debido al aumento de la cantidad de calorías ingeridas ya que cada vez consumen menos alimentos frescos hechos en casa y cada vez más se depende de productos industrializados (pre cocidos). La preferencia por este tipo de alimentos no es gratuita. La reducción en el tiempo disponible para la preparación es uno de los factores que Anderson et al (2013) ha considerado en el marco de una so-

\footnotetext{
${ }^{1}$ Para el 2012,37.2\% de adultos y 9.7\% entre niños menores de cinco años tenían este padecimiento. ${ }^{2}$ El cual ineludiblemente es un factor en el incremento de la prevalencia de la obesidad, al grado de que la Asociación Americana de Dietética establece el control del tamaño de la porción como una estrategia para el manejo del peso corporal (Rivera et al., 2012).
} 
ciedad en el que las mujeres se han incorporado más rápidamente al mercado laboral disminuyendo así el tiempo disponible a la preparación de alimentos. ${ }^{3}$

De igual modo, el cambio en la estructura de la familia: esto es, el caso de familias monoparentales, el incremento del desempleo masculino y la descomposición familiar y social pueden tener una explicación. En el caso de México, Heckman y Villarreal (2010) señalan que ha crecido el estrés familiar, ya que en México los cambios significativos en la estructura de las familias mexicanas están produciendo más hijos nacidos en entornos adversos.

Algunas de las tendencias adversas que han surgido en los últimos años son el aumento del número de niños nacidos en la pobreza, más nacimientos que se producen fuera del matrimonio, un aumento de los embarazos de adolescentes, más niños que viven con familias encabezadas por mujeres solteras, y más niños en hogares con un compañero ausente debido a la migración.

Todas estas tendencias indican una transformación de la familia en México, y su papel en el desarrollo del niño, que probablemente reduce la cantidad de recursos financieros disponibles para los niños y la calidad de la crianza de los hijos pues las madres que trabajan tienen una cantidad reducida de tiempo para estar con sus hijos, a menos que tengan acceso a las redes o sustitutos de cuidado de niños para su crianza. En este caso la mala alimentación que puede tener un niño de una familia mono parental debe ser de menor calidad y rica en alimentos procesados de fácil preparación y que tiene un alto contenido calórico que repercuten en la salud y el estado físico de los niños.

\footnotetext{
${ }^{3}$ Chou et al. (2004) profundizan en ese aspecto de las familias estadounidenses, al mencionar que desde finales de la década de los 1970 la tasa de obesidad en los adultos creció 50\%, y al igual que Anderson (op. cit.) encuentran la misma razón de tipo sociodemográfica.
} 


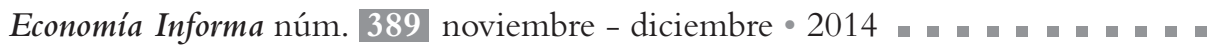

\section{Gráfica I}

Porcentaje de hogares encabezados por una mujer, 1984-2008

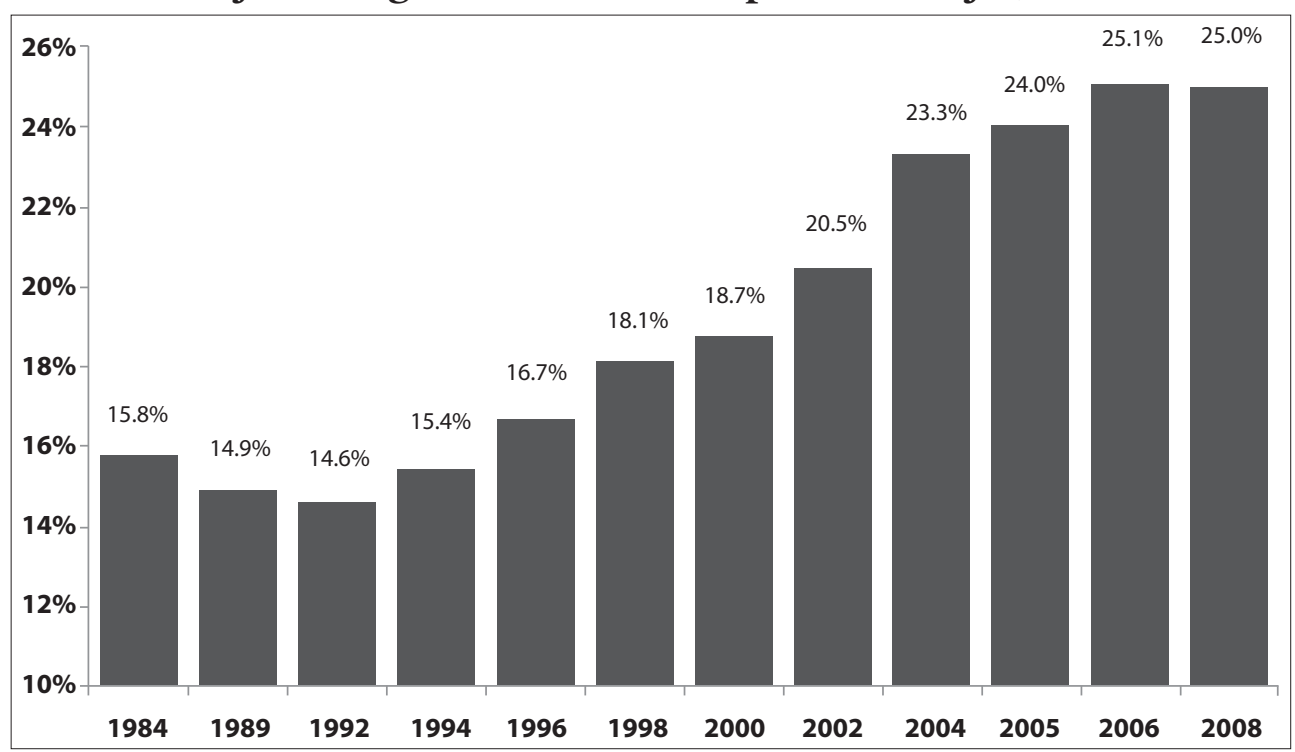

Fuente: Heckman y Villareal (2010).

La gran interacción entre los habitantes de México y Estados Unidos ha hecho que se adopten mutuamente hábitos alimenticios. De esta forma bien puede ser que la pandemia de obesidad que ya venían padeciendo los estadounidenses se incorporó a los problemas de salud pública de los mexicanos. De hecho uno de los efectos de la interacción de los hábitos alimenticios fue el incremento en la oferta de golosinas en México después de la firma del TLCAN, lo que abrió las posibilidades de la infancia para consumir de manera masiva alimentos ricos en calorías.

Para explorar esta hipótesis contrastamos los hábitos de consumo de los mexicanos a partir de los datos de la encuesta de ingreso-gasto de los hogares (ENIGH en 1992 y 1996), lo que arrojó un incremento sustancial (198\%), en la proporción del gasto destinado a la adquisición de dulces y golosinas, productos que por su composición y aporte calórico son altamente condicionantes en la prevalencia de sobrepeso y obesidad.

Este artículo se organiza de la siguiente manera. La primera sección presenta algunos hechos estilizados sobre el tema. En la segunda se analiza el problema en México. En la siguiente sección se presentan y se discuten los resultados que comparan datos de la ENIGH para 1992 y 1996. Al igual se presentan las conclusiones y algunas posibles líneas de análisis. 


\section{Algunos hechos estilizados}

De acuerdo a la OMs (2014) "El sobrepeso y la obesidad se definen como una acumulación anormal o excesiva de grasa que puede ser perjudicial para la salud".

Los niños con problemas de alimentación tienen muchas probabilidades de sobrepeso (Ortiz-Hernández y Cruz-Ángeles, 2005), y de convertirse en adultos obesos, en comparación con los niños sin sobrepeso. Adicionalmente tienen más probabilidades de sufrir diabetes y enfermedades cardiovasculares a edades más tempranas, que a su vez se asocian a un aumento de la probabilidad de muerte prematura y discapacidad" (oms, op cit).

El 44\% de los casos mundiales de diabetes, 23\% de cardiopatía isquémica y entre 7 y $41 \%$ de determinados cánceres son atribuibles al sobrepeso y la obesidad." (OMs, op cit).

La gráfica 2 muestra varios hechos interesantes. Para un periodo relativamente corto (1976-2006) se observa que en un grupo diverso de países:

1. Ha habido una clara tendencia al crecimiento de la obesidad, aun considerando a Japón, país cuyos hábitos alimenticios históricamente son más sanos que en el resto.

2. Desde 1994 esta tendencia se acentúa. Ningún país muestra una tendencia inversa.

3. Llama la atención que Estados Unidos siempre ha estado a la cabeza en la prevalencia.

4. Si bien México comienza en el cuarto lugar, detrás de Estados Unidos, Alemania e Inglaterra, la tendencia que observa es muy alarmante y cierra al final la brecha que tenia con el primer país. En el período 1988-1994 es de alrededor de 16 puntos, mientras que en la última observación (20052006) se cierra y la diferencia es de alrededor de 3 puntos.

5. A partir de un análisis más reciente, se observa que la prevalencia relativa continua creciendo al grado de que hacia el año 2010 México llego a ocupar el primer lugar en la OCDE, superando claramente a Estados Unidos, y casi duplicando el promedio de este organismo (11 vs 6.5\%). Ver gráfica 3 . 
Economía Informa núm. 389 noviembre - diciembre • 2014 - "

\section{Gráfica 2}

Tendencias en las prevalencias de obesidad en adultos en países seleccionados

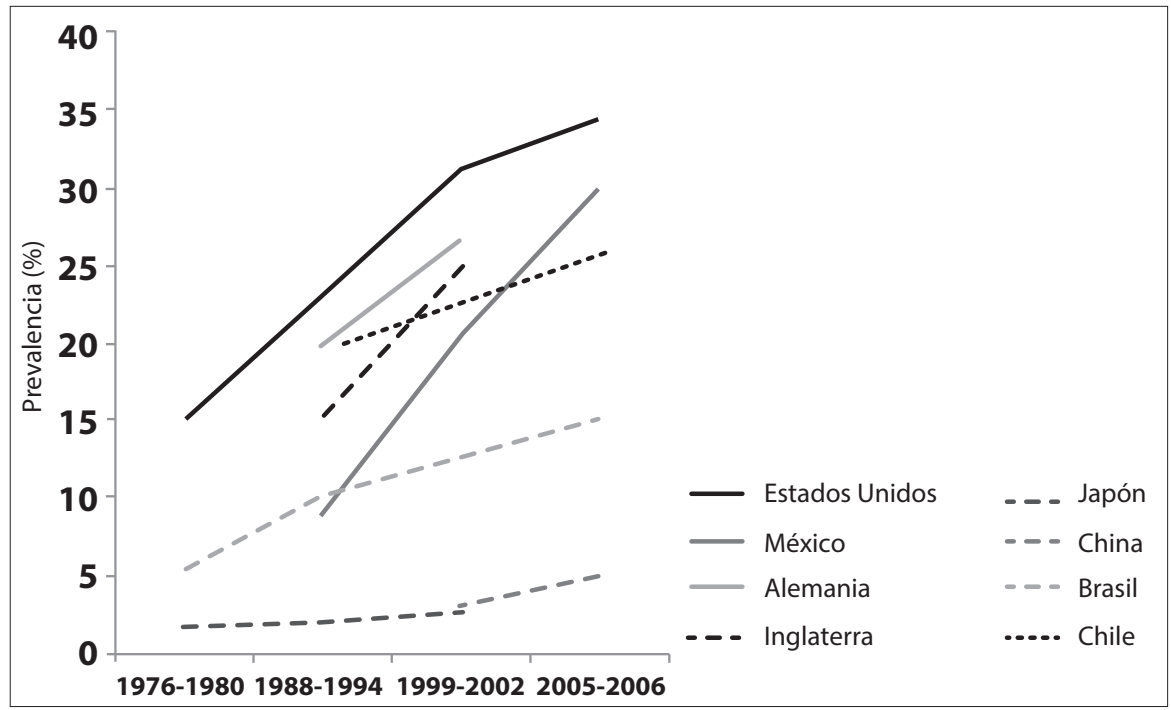

Fuente: López-Alarcón y Rodríguez-Cruz (2008).

\section{Gráfica 3}

Prevalencia de diabetes mellitus en población adulta entre 20 y 79 años de edad, 2010

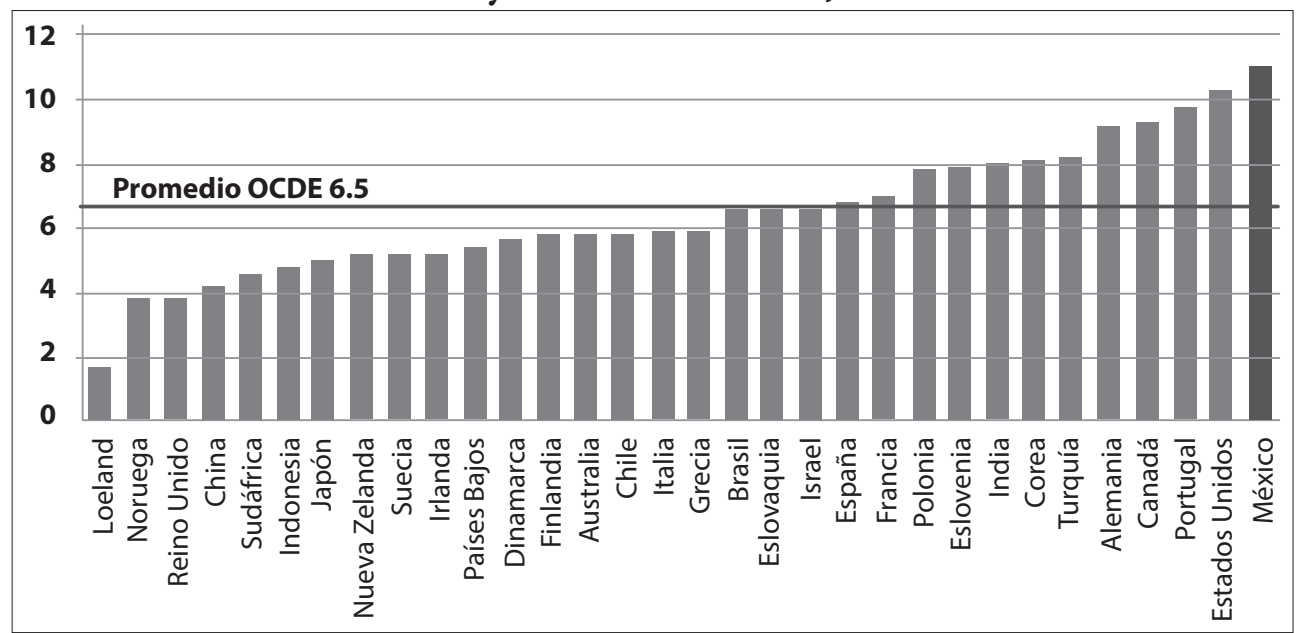

Fuente: Secretaría de Salud (2013). 
Mokdad et al (1999) revisaron para los Estados Unidos la relación condicional entre el aumento de la obesidad y la presencia de mayores casos de enfermedades crónico-degenerativas, para prever el potencial impacto en su sistema de salud. En el año 2012 en México se calculó el costo de atención por paciente diabético en US\$704 lo que llegó a representar 15\% de su presupuesto de salud para ese año y 17\% para 2013 (Pérez, 2011). En términos globales representó un gasto superior a los 105 mil millones de pesos (siete mil 784 millones de dólares) entre costos directos e indirectos, es decir, desde el tratamiento hasta el precio de las complicaciones relacionadas con la enfermedad, así como la mortalidad temprana (ICSs, 2013).

\section{La evolución reciente de la obesidad en México}

En la gráfica 4, se observa que la obesidad en México ha crecido dramáticamente particularmente en niños de 5 a 11 años, pero sobre todo en mujeres adultos (Villa et al., 2004). Si bien es un problema multifactorial, hay razones económicas -además de las ya mencionadas como consecuencia del ingreso al TLCAN- que no pueden despreciarse, como por ejemplo que el incremento en la ingesta de alimentos ricos en grasas y carbohidratos simples, los cuales por su relación aporte calórico-precio, pudieron ser más consumidos a manera de substitución como resultado del grave empobrecimiento que sufrió la población a raíz de la crisis de 1995.

\section{Gráfica 4}

\section{Prevalencia de sobrepeso y obesidad en México,} años seleccionados

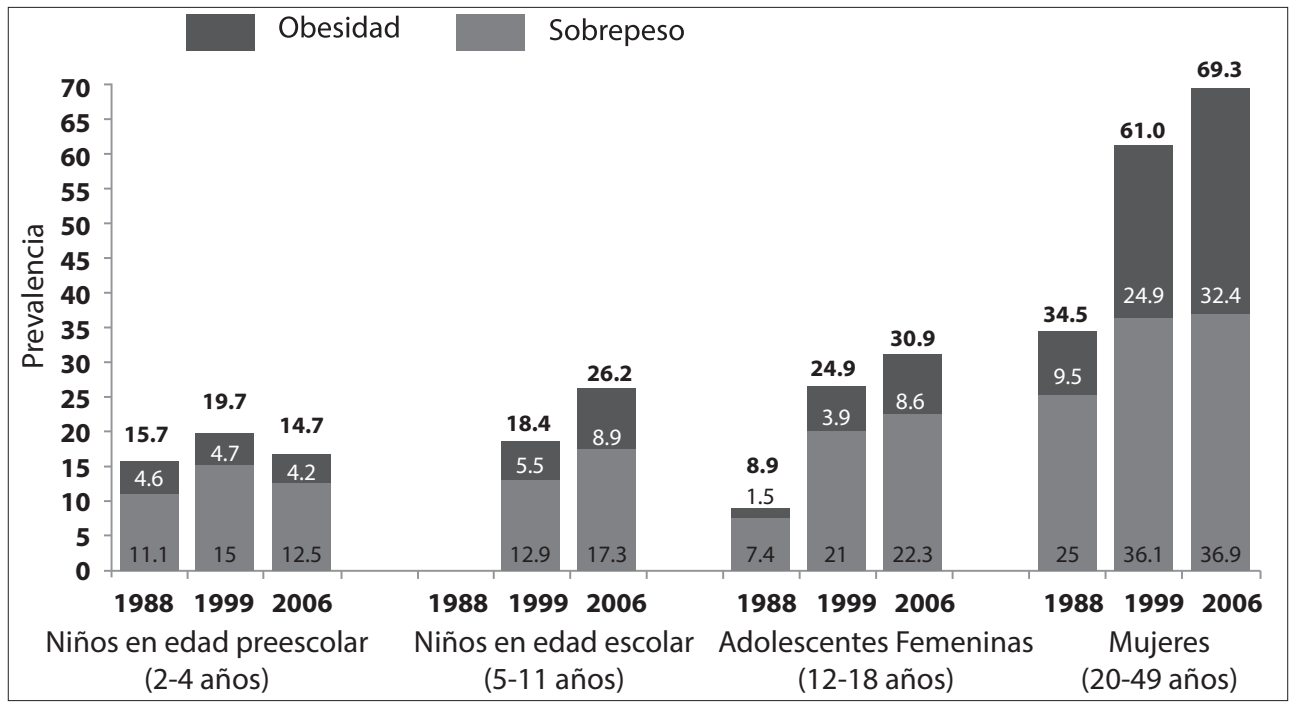

Fuente: INSP (2012). 
En efecto, además de los cambios alimenticios como consecuencia de los cambios sociodemográficos, de la descomposición (recomposición) de las familias y de la adopción de nuevos hábitos de consumo por la integración en Estados Unidos. La crisis de 1995 redujo el salario medio real que bien pudo provocar efectos sustitución en el consumo de una parte importante de los mexicanos hacia productos de bajo precio y de alto valor calórico. Ver gráfica 5.

Este aspecto es muy relevante, porque desde 1994 la variedad, oferta y disponibilidad de diversas golosinas creció de manera importante, a la vez que disminuyo relativamente su precio.

\section{Gráfica 5}

México: Salarios medios reales, 1980-2013

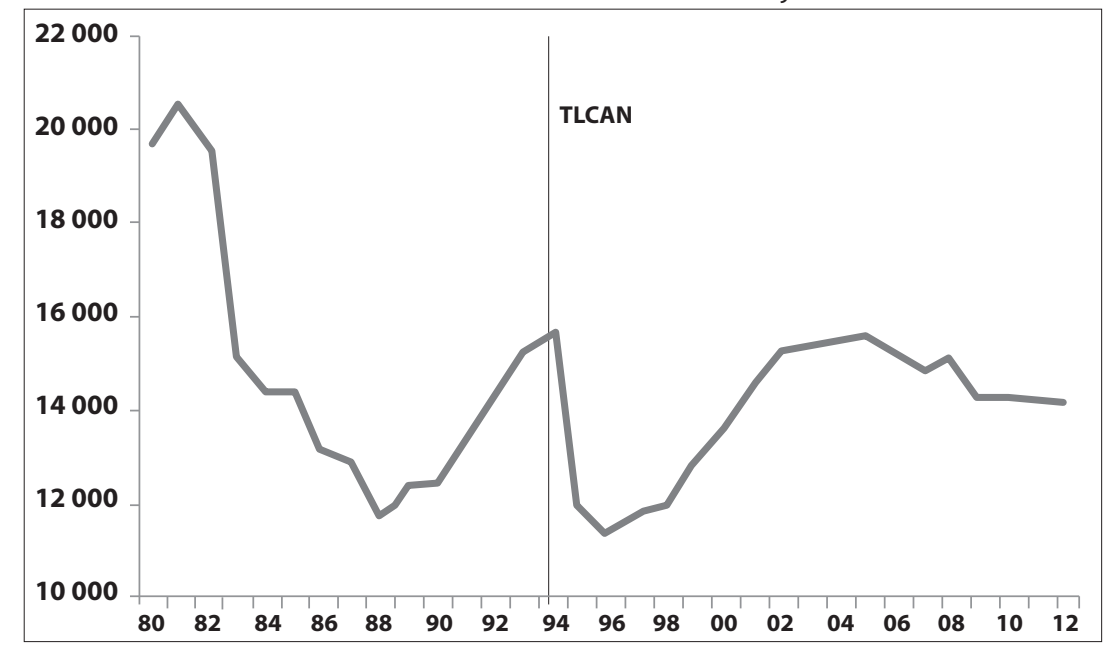

Fuente: INEGI (2012).

De igual manera, todos los indicadores de pobreza aumentaron de manera muy notable, lo cual apoya nuestra hipótesis de cambios de consumo. Ver gráfica 6. 


\section{Gráfica 6}

\section{México: Evolución de la pobreza por Ingresos,}

Millones de Personas 1992-2010

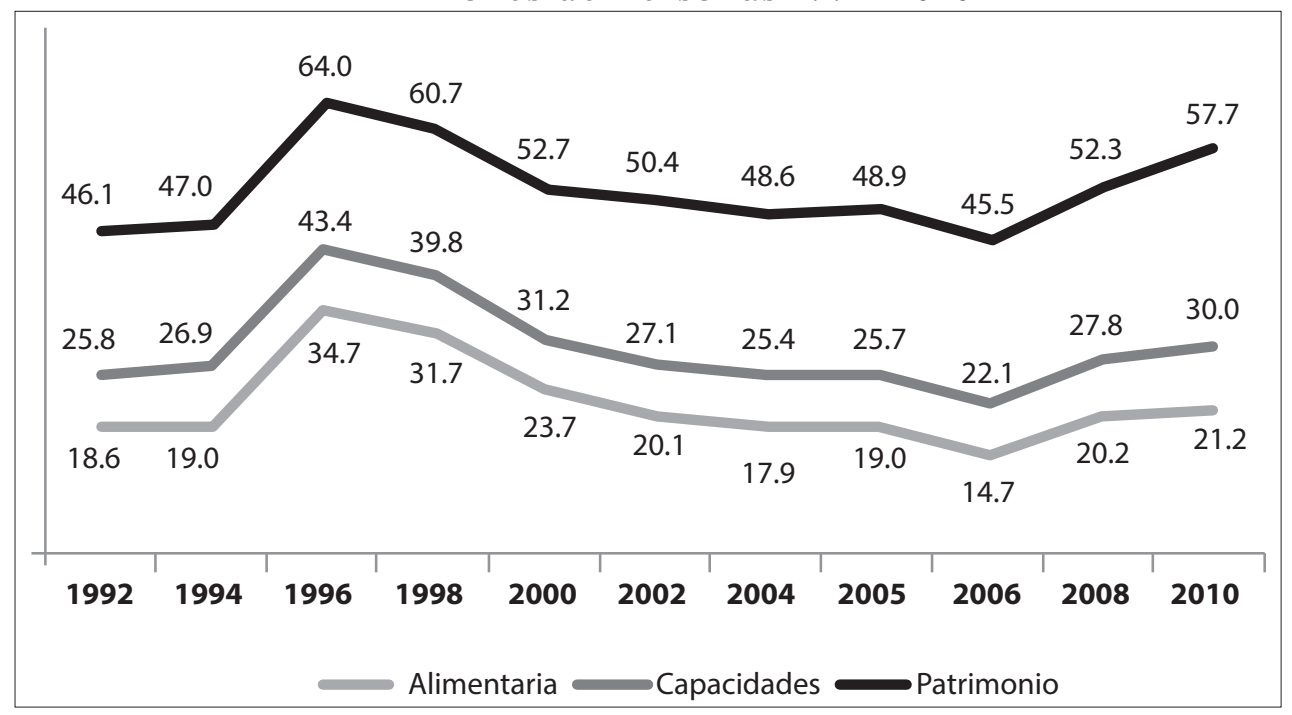

Fuente: Sedesol (2012).

Si bien el problema es general, el segmento de la población más preocupante es el de los niños por ser altamente susceptibles a impulsos en el entorno para gestar cambios dentro de su dieta que les llevaría a incrementar los riesgos de padecer sobrepeso u obesidad.

Dejando de lado el dramático efecto ingreso de la crisis de 1995 que bien pudo haber contribuido de manera importante, consideramos que la apertura comercial creó un cambio en la oferta con nuevas técnicas de mercadeo, que hicieron uso intensivo de las propiedades del consumo impulsivo, ${ }^{4}$ como ocurrió en otros países de América Latina (Castillo y Romo, 2006).

Swinburn et al (2011) proponen que el incremento de la obesidad en casi todos los países puede deberse a cambios del sistema alimentario mundial, que está produciendo comida más procesada, a precios más asequibles y comercializada con más eficacia que antes.

\footnotetext{
${ }^{4} \mathrm{O}$ consumo compulsivo que se define como "Aquél que uno tiene que realizar para satisfacer las necesidades biológicas, culturales y situacionales y que se da cuando uno cuenta con pocos recursos para atenderlas o no tiene alternativas para elegir. En este caso, cuando la persona realiza sus compras, siempre busca lo que es más barato, aunque el producto sea de mala calidad. Lo que importa es llevarse a casa la mayor cantidad posible de mercadería. Aquí, las personas no están preocupadas por la calidad del producto ni por las marcas famosas, sino en maximizar la cantidad de lo que podrían comprar con el mismo dinero" (Mance, 2003: 80).
} 
Economía Informa núm. 389 noviembre - diciembre • 2014 - "

Esa nueva oferta ha incorporado crecientemente golosinas que por su composición rica en grasas y azúcares funcionan como poderosas fuentes energéticas de recompensa neurobiológica (Castillo y Romo, 2006) y de reducción del nombre (sensación de saciedad).

Algunas de estas nuevas técnicas de mercadeo usaron el crecimiento de las porciones para influir en mayor consumo (Wansink, 2006); hecho que se ha documentado en Estados Unidos como un determinante de la pandemia de obesidad, ya que desde la década de 1970 los productores han incrementado sistemáticamente el tamaño de las porciones de manera paralela al incremento del peso corporal promedio de los Estados Unidos (Young y Nestle, 2002 y 2007).

El efecto de las nuevas técnicas que asocian el consumo impulsivo y el incremento en las porciones tiene efectos focalizados dentro de la población infantil (Krebs et al., 2006) lo que sumado a los ya mencionados anteriormente crea un mercado cautivo y sistemático desde la infancia.

\section{Análisis comparado del gasto de consumo}

El gasto del hogar es el desembolso que se realiza para atender las necesidades y cumplir con sus compromisos. El gasto corriente da cuenta de los gastos realizados regularmente en el hogar, a precios de mercado, para la adquisición de su canasta de consumo, además del gasto regular no destinado al consumo.

Una razonable aproximación de los hábitos de consumo de los mexicanos se encuentra en la Encuesta Nacional de Ingreso-Gasto de los Hogares (ENIGH, 2012) que resulta de aplicar alrededor de 300000 encuestas.

La ENIGH presenta un tabulado de gasto corriente desagregado en gasto corriente monetario y gasto corriente no monetario que se agrupa en las siguientes categorías, ver cuadro 1. 


\section{Cuadro I}

México: lista de categorías de la ENIGH, 2013

\begin{tabular}{|l|c|}
\hline Categorías & Número de Productos \\
\hline A. Alimentos, bebidas y tabaco & 205 \\
\hline B. Transporte público & 7 \\
\hline C. Limpieza y cuidado de la casa & 24 \\
\hline D. Artículos para el cuidado personal & 22 \\
\hline E. Educación, cultura y recreación & 25 \\
\hline F. Comunicaciones y servicios para vehículos & 10 \\
\hline G.Vivienda y servicios de conservación & 24 \\
\hline H. Prendas de vestir, calzado y accesorios & 64 \\
\hline I. Cristalería, blancos y utensilios domésticos & 24 \\
\hline J. Cuidados de la salud & 43 \\
\hline K. Enseres domésticos y mantenimiento de la vivienda & 31 \\
\hline L. Artículos de esparcimiento & 24 \\
\hline M. Transporte & 16 \\
\hline N. Otros gastos & 15 \\
\hline Total & 534 \\
\hline
\end{tabular}

Fuente: ENIGH (2013).

Nota: no se incluyen: erogaciones financieras y de capital, ingresos netos del hogar y percepciones financieras y de capital.

Para efectos de esta investigación se procedió a concentrar a los subgrupos por homoclave ${ }^{5}$ de la categoría "A", que estructura el consumo de bienes alimenticios que contuvieran toda la información necesaria para nuestro análisis y de esa manera hacer un acercamiento sobre el cambio en los hábitos de consumo de los mexicanos. Elegimos dos encuestas que de manera indirecta muestran este hipotético cambio por el TLCAN que son los de: 1992 y 1996.

Debido a la enorme dificultad de medición, se asumió como hipótesis la estabilidad de los precios relativos, ${ }^{6}$ y se midió el peso relativo del gasto de los grupos construidos en el consumo para cada año seleccionado, cuadro 2.

\footnotetext{
${ }^{5}$ Basada en Sistema de Clasificación Industrial de América del Norte (SCIAN) 2004, versión hogares.

${ }^{6}$ Precio de un bien en relación a los demás.
} 


\section{Cuadro 2}

Porcentajes de gasto en alimentos. ENIGH, 1992-1996

(en porcentajes)

\begin{tabular}{|c|c|c|}
\hline Rótulos de fila & 1992 & 1996 \\
\hline Aves & 6.17 & 6.37 \\
\hline Azúcares y mieles & 1.51 & 0.05 \\
\hline Bebidas & 4.70 & 4.66 \\
\hline Carne de puerco & 2.98 & 2.96 \\
\hline Carne de res & 10.22 & 9.03 \\
\hline Cereales & 14.31 & 16.53 \\
\hline Alimentos preparados & 3.04 & 1.76 \\
\hline Dulces & 0.56 & 1.67 \\
\hline Frutas y verduras & 16.54 & 15.26 \\
\hline Huevo & 3.36 & 4.70 \\
\hline Otros alimentos & 34.62 & 34.96 \\
\hline Pescados y mariscos & 1.98 & 2.04 \\
\hline Total General & 100.00 & 100.00 \\
\hline
\end{tabular}

Como ya se mencionó, se tomaron dos observaciones inmediatas alrededor de la entrada en vigor del TLC (1992 y 1996) que permiten contrastar con claridad nuestra hipótesis. Si bien el gasto en proteínas animales no cambió sustancialmente, si se observan incrementos relevantes en el gasto de cereales y sobretodo en dulces de 0.56 a $1.67 \%$. Este último representó un incremento de 196\% en tan sólo cuatro años.

\section{Conclusiones}

Se calcularon los pesos relativos de "grupos de alimentos" de la ENIGH para dos años representativos pre y post entrada en vigor la firma del tLCAN. Se compararon esos pesos relativos que incorporan precios y cantidades. Bajo el supuesto de que el "vector de precios relativos" se mantuvo relativamente constante, los cambios en los pesos muestran los efectos del cambio en el consumo de cantidades.

Apreciamos una relativa constancia entre las proporciones de consumo en prácticamente todos los rubros, las cuales hemos de advertir que no están estratificadas por nivel de ingreso debido a que pertenecen a toda la encuesta. 
El único incremento significativo que apreciamos está en el rubro de "cereales" y sobre todo en el de "dulces". El incremento en este tipo de alimentos, independientemente del aporte calórico el cual de por sí ya es elevado, es condicionante para el incremento de peso, como lo proponen Gazzaniga, y Burns (1993).

Muy probablemente estos nuevos dulces cubran dos características: la primera de ellas es que la presentación haya crecido en el tiempo, el segundo de ellos es que estén endulzados con fructosa, ${ }^{7}$ particularmente proveniente de jarabe de maíz y no de azúcar de caña.

El jarabe de maíz contiene beneficios para la industria como ser más dulce que el azúcar y con beneficios como reducción de costos para la industria que cada vez se enfrenta a una mayor demanda de alimentos baratos como bebidas carbonatadas, panadería, frutas enlatadas, mermeladas y jaleas, entre otros (Hanover y White, 1993).

De hecho Gross et al (2004) apuntan a que desde la década de los sesenta en los Estados Unidos el consumo de jarabe de maíz ha reemplazado a otros edulcorantes y se ha añadido a la dieta en otras formas de carbohidratos como los granos enteros con fibra, lo que bien ha podido ser una de las condicionantes para explicar la epidemia de sobrepeso y obesidad, aunado al problema de diabetes mellitus.

El problema con el consumo del jarabe de maíz es a nivel metabólico porque "puede impactar los lípidos de la sangre y la resistencia de la insulina y por ende puede incrementar los factores de riesgo para enfermedades del corazón y la diabetes tipo 2" (Dahl et al, 2014). Asimismo Havel (2002) refiere que el consumo fructosa, que no inician la secreción de insulina, puede reducir los niveles de leptina y de conducir a comer en exceso. He allí otro punto adicional para el uso de este jarabe como aditivo en varios alimentos.

Rivera et al $(2012,58)$ apuntan que por lo menos hace 30 años el azúcar añadida mediante el jarabe de alta fructuosa se ha asociado a una mayor ganancia de peso. El consumo de este edulcorante no es dañino per se (Dahl et al, op. cit.), lo que buscamos en este trabajo es notar que el cambio en los hábitos de alimentación que incluyeron mayores porciones e incremento en la oferta de alimentos industrializados contenían aditivos a los que los mexi-

${ }^{7}$ Este edulcorante ha tenido un gran crecimiento en México, según datos del Centro de Estudios de las Finanzas Públicas la importación de fructuosa pura ha pasado de US \$10 millones en 1990, antes de la firma del TLCAN, a US \$4,999 millones en el 2004, lo que representó un consumo de 6.5 millones de toneladas (CEFP, 2005: 5). 
Economía Informa núm. 389 noviembre - diciembre • 2014 - "

canos no estábamos acostumbrados fue uno de los detonantes de la epidemia de sobrepeso y obesidad.

\section{Bibliografía}

Anderson, P. M., Butcher, K. F., \& Levine, P. B. (2003), Maternal employment and overweight children. Journal of health economics, 22(3), 477-504.

ввС. (2014). ¿Ha ayudado el impuesto a los refrescos a reducir la epidemia de obesidad? http://noticias.prodigy.msn.com/internacional/\%C2\%BFha-ayudado-elimpuesto-a-los-refrescos-a-reducir-la-epidemia-de-obesidad

Booth, K. M., Pinkston, M. M., \& Poston, W. S. C. (2005), Obesity and the built environment. Journal of the American Dietetic Association, 105(5), 110-117.

Castañeda, J. (2014). Más TLC. Nexos. http://www.nexos.com.mx/?p=15680

Castillo D, C., \& Romo M, M. (2006), Las golosinas en la alimentación infantil. Revista chilena de pediatría, 77(2), 189-193.

CEFP (2005), El Impacto de las Importaciones de Fructosa en la Industria Azucarera http://www.cefp.gob.mx/intr/edocumentos/pdf/cefp/cefp0222005.pdf

Chou, S. Y., Grossman, M., \& Saffer, H. (2004), An economic analysis of adult obesity: results from the Behavioral Risk Factor Surveillance System. Journal of health economics, 23(3), 565-587.

Cutler, D., Glaeser, E., \& Shapiro, J. (2003), Why have Americans become more obese? (No. w9446). National Bureau of Economic Research.

Dahl, W. J., Foster, L., \& Owen, R. J. (2014), Los hechos acerca de la fructosa. https://edis.ifas.ufl.edu/pdffiles/FS/FS18400.pdf

ENIGH (2012), Encuesta Nacional Ingreso Gasto de los Hogares. http://www. inegi.org.mx/est/contenidos/Proyectos/encuestas/hogares/regulares/enigh/

Gazzaniga, J. M., \& Burns, T. L. (1993), Relationship between diet composition and body fatness, with adjustment for resting energy expenditure and physical activity, in preadolescent children. The American journal of clinical nutrition, 58(1), 21-28.

Gross, L. S., Li, L., Ford, E. S., \& Liu, S. (2004), Increased consumption of refined carbohydrates and the epidemic of type 2 diabetes in the United States: an ecologic assessment. The American journal of clinical nutrition, 79(5), 774-779.

Heckman, J. J., \& Villarreal, C. (2010), Policies to promote growth and economic efficiency in mexico (No. w16554). National Bureau of Economic Research. 
Hernandez, B., Gortmaker, S. L., Colditz, G. A., Peterson, K. E., Laird, N. M., \& Parra-Cabrera, S. (1999), Association of obesity with physical activity, television programs and other forms of video viewing among children in Mexico City. International journal of obesity and related metabolic disorders: journal of the International Association for the Study of Obesity, 23(8), 845-854.

Havel, P. J. (2002), Control of energy homeostasis and insulin action by adipocyte hormones: leptin, acylation stimulating protein, and adiponectin. Current opinion in lipidology, 13(1), 51-59.

Hanover, L. M., \& White, J. S. (1993), Manufacturing, composition, and applications of fructose. The American journal of clinical nutrition, 58(5), 724S-732S.

ICSS. (2013), Diabetes cuesta a México 105 MMDP. Instituto Carlos Slim para la Salud. http://www.salud.carlosslim.org/diabetes-cuesta-a-mexico-105-mmdp-2/

INSP. (2012), La Obesidad en México. Instituto Nacional de Salud Pública. http:// www.uv.mx/nutri-xal/files/2013/02/La-obesidad-en-Mexico.pdf

Jeffery, R. W., Baxter, J., McGuire, M., \& Linde, J. (2006), Are fast food restaurants an environmental risk factor for obesity?. International Journal of Behavioral Nutrition and Physical Activity, 3(1), 2.

Krebs, N. F., Himes, J. H., Jacobson, D., Nicklas, T. A., Guilday, P., \& Styne, D. (2007), Assessment of child and adolescent overweight and obesity. Pediatrics, 120 (Supplement 4), S193-S228.

López-Alarcón, M. G., \& Rodríguez-Cruz, M. (2008), Epidemiología y genética del sobrepeso y la obesidad: Perspectiva de México en el contexto mundial. Boletín médico del Hospital Infantil de México, 65(6), 421-430.

Mokdad, A. H., Serdula, M. K., Dietz, W. H., Bowman, B. A., Marks, J. S., \& Koplan, J. P. (1999), The spread of the obesity epidemic in the United States, 1991-1998. Jama, 282(16), 1519-1522.

Mance, E. A. (2003), Consumo solidário. A outra economia. Porto Alegre: Veraz, 45.

OMS (2014), Organización Mundial de la Salud, Centro de Prensa, Obesidad y Sobrepeso, Nota descriptiva 311. http://www.who.int/mediacentre/factsheets/ fs $311 /$ es/

OPS. (2014), Organización Panamericana de la Salud. Obesidad. http://www.paho. org $/ \mathrm{mex} /$ index.php?option $=$ com_content $\&$ view $=$ category\&id $=824$

Ortiz-Hernández, L., \& Cruz-Ángeles, L. I. (2005), Asociación del crecimiento físico con la composición corporal en escolares de Xochimilco. Boletín médico del Hospital Infantil de México, 62(6), 428-442. 
Economía Informa núm. 389 noviembre - diciembre • 2014

Pérez, V. (2011), Cada paciente con diabetes le cuesta 708 dólares al año a México. CNN México. http://mexico.cnn.com/salud/2011/06/13/cada-paciente-condiabetes-le-cuesta-708-dolares-al-ano-a-mexico

Rashad, I., Grossman, M., \& Chou, S. Y. (2005), The super size of America: an economic estimation of body mass index and obesity in adults (No. w11584). National Bureau of Economic Research.

Rivera, J., Hernández, M., Aguilar, C. A., Vadillo, F., \& Murayama, C. (2012), Obesidad en México: Recomendaciones para una política de Estado, UNAM.

Secretaría de Salud. (2013), Estrategia Nacional para la Prevención y el Control del Sobrepeso, la Obesidad y la Diabetes. http://promocion.salud.gob.mx/dgps/ descargas1/estrategia/Estrategia_con_portada.pdf

Swinburn, B. A., Sacks, G., Hall, K. D., McPherson, K., Finegood, D. T., Moodie, M. L., \& Gortmaker, S. L. (2011), The global obesity pandemic: shaped by global drivers and local environments. The Lancet, 378(9793), 804-814.

Villa, A. R., Escobedo, M. H., \& Méndez-Sánchez, N. (2004), Estimación y proyección de la prevalencia de obesidad en México a través de la mortalidad por enfermedades asociadas. Gac Méd Méx, 140(2), S21-26.

Wansink, B. (1996), Can package size accelerate usage volume? The Journal of Marketing, 1-14.

Young, L. R., \& Nestle, M. (2002), The contribution of expanding portion sizes to the US obesity epidemic. American journal of public health, 92(2), 246-249.

(2007), Portion sizes and obesity: responses of fast-food companies. Journal of public health policy, 28(2), 238-248. 